\section{Perfil dos microrganismos isolados no trato urinário após sondagem vesical em cirurgia ginecológica}

\section{Profile of micro-organisms found in urocultures after urinary catheterization in patients undergoing elective gynecological surgery}

Sônia Cristina Hinrichsen 1

Melania Maria Ramos Amorim²

Alex Sandro Rolland Souza ${ }^{3}$

Aurélio Costa 4

Maria Gabriela M.L. Hinrichsen 5

Sylvia Lemos Hinrichsen ${ }^{6}$

1-5 Centro de Atenção à Mulher. Instituto de Medicina Integral Professor Fernando Figueira, IMIP. Rua dos Coelhos, 300. Recife, PE, Brasil. CEP: 50.070-550 E-mail: melamorim.@uol.com.br 2 Núcleo de Ensino e Pesquisa e Assistência em Infectologia. Universidade Federal de Pernambuco. Recife, PE, Brasil.

\section{Resumo}

Objetivos: determinar os microrganismos freqüentemente encontrados nas uroculturas após sondagem vesical e o perfil de sensibilidade aos antibióticos em mulheres submetidas a cirurgia ginecológica eletiva.

Métodos: estudo de corte transversal em mulheres submetidas a cirurgia ginecológica com sondagem vesical, no Instituto de Medicina Integral Professor Fernando Figueira (IMIP), em Recife, Pernambuco, Brasil, de janeiro a maio de 2007. As uroculturas foram coletadas em dois periodos: até $24 \mathrm{~h}$ da retirada do cateter e após sete/10 dias.

Resultados: amostras de urina foram colhidas em 249 mulheres. Encontrou-se 23,6\% $(n=46)$ de uroculturas positivas com até $24 \mathrm{~h}$ da retirada da sonda e $11,1 \%(n=25)$ com sete 10 dias após sondagem vesical. Não foi observada diferença significativa em relação aos microrganismos, quando se compararam as duas uroculturas. A Klebsiella spp. foi o principal microrganismo em ambas uroculturas (até $24 h=47,8 \%$ vs sete $/ 10$ dias $=44,0 \% ; p=0,76$ ), seguido de Escherichia coli e outros Gram-negativos. Os antibióticos amicacina, cefepima, ciprofloxacina, meropenem e ticarcilina-clavulanato foram os que apresentaram sensibilidade igual ou superior a $75 \%$ para os principais microrganismos isolados.

Conclusões: Klebsiella spp. foi o microrganismo mais encontrado nas uroculturas após sondagem vesical em cirurgias ginecológicas e o antibiótico com sensibilidade igual ou maior a 95\% com via de administração oral foi a ciprofloxacina.

Palavras-chave Bacteriuria, Cateterismo urinário, Infecções urinárias, Resistência a drogas, Agentes antibacterianos 


\section{Introdução}

A infecção do trato urinário (ITU) caracteriza-se pela presença de agentes infecciosos e invasão tissular em qualquer parte do trato urinário (urina, bexiga e rins), podendo ser acompanhada ou não por sintomas.1,2 Desta forma, a colonização bacteriana quando ocorre na urina é conhecida como bacteriúria, sendo também classificada em sintomática ou assintomática. ${ }^{3}$

A bacteriúria assintomática é definida mais precisamente como o isolamento de bactérias na urina em quantidade maior ou igual a $10^{5}$ unidade formadora de colônia por mililitro (UFC/mL), sem sinal ou sintoma local ou sistêmico.3-6 Abaixo desse valor, a bacteriúria pode significar contaminação, contudo a maioria dos microrganismos isolados é típica da ITU, como a E. coli, outros Gram-negativos ou Staphylococcus saprophyticus. ${ }^{7}$

A cateterização vesical é uma condição que predispõe à bacteriúria, que é geralmente assintomática, mas que pode tornar-se sintomática, com risco de bacteremia, estando associada ao aumento da incidência de ITU.1,3,8,9 Desta forma, sua introdução deve ser realizada segundo técnicas rígidas de anti-sepsia. $8,10 \mathrm{O}$ risco de adquirir uma infecção do trato urinário relacionada à cateterização depende principalmente do método e da duração da cateterização, além da qualidade do cateter e da susceptibilidade do paciente.10-12 As taxas de infecção variam em torno de $8 \%$ após uma única cateterização por período breve 13 e aumentam de $5 \%$ a $8 \%$ a cada dia de cateterização. 14 Estudos demonstram que em torno de 10 a $20 \%$ dos pacientes cateterizados e mantidos em drenagem fechada podem apresentar ITU.8,15 Merecem atenção especial as cirurgias ginecológicas que utilizam rotineiramente o cateterismo vesical para diminuir a dor pélvica e a retenção urinária. 16 Por não se conhecer o real benefício dessa prática, a orientação é de limitar a sondagem vesical, 10 uma vez que o risco estimado para ocorrência de infecção urinária pode ser cinco vezes maior. ${ }^{17}$

As infecções urinárias associadas à cateterização vesical podem ser causadas por Escherichia coli, Klebsiella spp., Proteus mirabilis, espécies de Enterococcus, Pseudomonas spp., Enterobacter spp., Serratia spp. e Candida spp.9,18 Muitos desses microrganismos fazem parte da flora endógena do intestino e podem contaminar o trato urinário pela proximidade anatômica ou veiculado pelos profissionais de saúde. A presença de patógenos como Serratia marcescens e Burkholderia cepacia tem especial importância, porque pode indicar a existência de uma fonte exógena para essa aquisição. ${ }^{15}$ A E. coli é o microrganismo mais freqüente das ITU, estando presente em aproximadamente $85 \%$ das infecções comunitárias e $50 \%$ das infecções hospitalares. 18,19 Outras enterobactérias Gram-negativas, incluindo Proteus spp., Klebsiella spp., Enterobacter spp. e Pseudomonas spp., estão implicadas na etiologia da ITU, ocorrendo em pacientes com doenças associadas ou nas infecções hospitalares complicadas. Além disso, estudos sugerem que os microrganismos mais associados às infecções urinárias devido ao cateterismo vesical, como E. coli, Pseudomonas spp., Proteus spp. e Klebisiella spp., apresentam alta resistência aos antibióticos mais utilizados, como a ampicilina, gentamicina e sulfametoxazol-trimetoprim.20,21

O presente estudo foi realizado com o objetivo de determinar a freqüencia dos microrganismos isolados no trato urinário após sondagem vesical e seus perfis de sensibilidade aos antibióticos nas cirurgias ginecológicas.

\section{Métodos}

Realizou-se um estudo observacional, analítico, do tipo corte transversal, de base hospitalar, para determinar a freqüência de microrganismos isolados no trato urinário e seus perfis de sensibilidade, após sondagem vesical em mulheres submetidas a cirurgia ginecológica eletiva no Instituto de Medicina Integral Professor Fernando Figueira (IMIP), em Recife, Pernambuco, Brasil.

O estudo foi desenvolvido na enfermaria de ginecologia do IMIP, no período de janeiro a maio de 2007. As mulheres selecionadas encontravam-se acima de 13 anos e foram submetidas a cirurgia ginecológica eletiva com cateterização vesical. Excluíram-se as mulheres antes da menarca, as que se encontravam fazendo uso de antibioticoterapia e aquelas que apresentaram a urocultura positiva antes da sondagem vesical.

O tamanho amostral foi calculado usando o software Statcalc do Epi-Info 3.4.1. Prevendo-se uma freqüência de infecção do trato urinário de $10 \%, 13,22$ com uma precisão de $5 \%$ e um intervalo de confiança de $95 \%$, foi estimado um número de 216 mulheres como representativo da amostra. Considerando eventuais perdas em torno de $20 \%$, selecionaram-se 260 pacientes, das quais 11 foram excluídas, ficando para análise final 249. A exclusão de duas ocorreu por início de antibiótico curativo, uma por extravio das uroculturas e oito por urocul- 
tura positiva antes da colocação da sonda vesical.

As variáveis analisadas foram o tipo da cirurgia, uso de antibiótico profilático, duração da sondagem vesical, presença de sintomas associados (febre e/ou dor e/ou aumento da freqüência urinária e/ou disúria), o microrganismo isolado e o resultado do antibiograma nas amostras de urina coletadas com até 24 horas depois da retirada da sonda e 7/10 dias da sondagem vesical.

A bacteriúria foi definida pela presença de 100.000 ou mais unidades formadoras de colônia por mililitro (UFC/mL), após o isolamento da bactéria ou fungo nas culturas de urina. 5,6

A sondagem vesical foi realizada com um cateter de Foley de duas vias, do número 12 ao 18, seguindo técnica asséptica padronizada pela Comissão de Controle de Infecção Hospitalar (CCIH) do IMIP, colocada por profissional de saúde tecnicamente capacitado. O procedimento foi realizado após lavagem das mãos e colocação de luvas estéreis. A mulher encontrava-se em decúbito dorsal e pernas afastadas, seguindo-se anti-sepsia da região perineal, periuretral com solução anti-séptica. O balão do cateter foi preenchido com 10 a $20 \mathrm{~mL}$ de água destilada estéril, mantendo o sistema de drenagem fechado.

Uma coleta de urina foi realizada antes da cirurgia para exclusão de mulheres com uroculturas positivas. As uroculturas até 24 horas após a retirada da sonda vesical foram coletadas ainda durante a internação hospitalar. As uroculturas com sete/10 dias da sondagem vesical foram colhidas na consulta ambulatorial. As coletas de urina foram realizadas pelas próprias pacientes após instruções, desprezando-se o primeiro jato urinário e dirigindo-se o segundo jato para o recipiente estéril. O tempo de permanência da sonda seguia orientação do médico assistente, não havendo influência do pesquisador responsável. O tratamento antibiótico foi iniciado apenas na presença de sintomatologia.

A análise microbiológica foi realizada no laboratório de microbiologia IMIP. O semeio da cultura foi realizado com a alça calibrada de $1 \mu \mathrm{L}$ (multiplicador 1.000), sendo sua diluição de 1:1.000. Os meios de cultura para semeadura foram o ágar Cysteine Lactose Eletrolyte-Deficient Médium (CLED), para crescimento de germes Gram-negativos, Gram-positivos, inclusive enterococos, e o ágar de McConkey e ágar sangue (meio de base Casoy com 5\% de sangue de carneiro desfibrinado), quando necessário. $\mathrm{O}$ meio de Sabouraud foi utilizado para a pesquisa de leveduras. O antibiograma foi realizado pelo método de Kirby-Bauer22 quando observado crescimento bacteriano, conforme normas estabelecidas pelo National Committee for Clinical Laboratory Standards (NCCLS). ${ }^{5}$

A análise estatística foi realizada com o programa de domínio público Epi-Info versão 3.4.1. Calcularam-se as freqüências das variáveis categóricas e a comparação das freqüências dos microrganismos entre as duas uroculturas foi realizada pelo teste exato de Fisher, considerando-se um nível de significância de $95 \%$.

Este estudo foi aprovado pelo Comitê de Ética em Pesquisa do IMIP, Parecer n.904 de 17 de novembro de 2006, sendo que todas as mulheres assinaram o Termo de Consentimento Livre e Esclarecido após terem sido informadas da natureza do estudo.

\section{Resultados}

Incluíram-se no estudo 249 mulheres submetidas a cirurgias ginecológicas com sondagem vesical, sendo colhidas 195 uroculturas com até 24 horas da retirada da sonda e 226 uroculturas sete/10 dias após sondagem vesical. Dessas, 23,6\% $(n=46)$ foram positivas logo na primeira coleta e $11,1 \%(n=25)$ resultaram positivas com sete/10 dias da sondagem vesical, sendo $2,4 \%(n=6)$ de bacteriúria sintomática. O tratamento com antibióticos foi iniciado apenas nas mulheres sintomáticas $(n=6)$, sendo colhida a segunda amostra antes de instituída a antibioticote-

\section{Tabela 1}

Microrganismos isolados nas uroculturas das mulheres submetidas a cirurgia ginecológica após sondagem vesical em um hospital escola do nordeste do Brasil, em 2007.

\begin{tabular}{lccccc}
\hline Microrganismo** & $\begin{array}{c}\text { Urocultura até } \\
\text { 24 horas }(\mathbf{n}=\mathbf{4 6})\end{array}$ & $\begin{array}{c}\text { Urocultura sete/10 } \\
\text { dias }(\mathbf{n}=\mathbf{2 5})\end{array}$ & $\boldsymbol{p}^{*}$ \\
\cline { 2 - 5 } & $\mathrm{n}$ & $\%$ & $\mathrm{n}$ & $\%$ & \\
\cline { 2 - 5 } & 22 & 47,8 & 11 & 44,0 & 0,76 \\
Klebsiella spp. & 12 & 26,1 & 6 & 24,0 & 0,85 \\
Escherichia coli & 8 & 17,4 & 5 & 20,0 & 0,51 \\
Outros Gram- Negativos & $3 * * *$ & 6,5 & - & - & 0,27 \\
Fungos (Candida) & $2 * * * *$ & 4,4 & - & - & 0,42 \\
Estafilococos Coagulase- & & & & & \\
$\quad$ Negativos & 2 & 4,4 & 2 & 8,0 & 0,44 \\
Streptococcus do grupo B & 1 & 2,2 & - & - & 0,65 \\
P. aeruginosa & - & - & 1 & 4,0 & 0,35 \\
Staphylococcus saprophyticus & - & & & \\
\hline
\end{tabular}

* Teste exato de Fisher; ** Uma mulher pode ter um ou mais tipos de germe; *** Microrganismos isolados nas três mulheres: Candida spp. e E. coli; **** Microrganismos isolados em uma mulher: Estafilococo Coagulase-Negativo e Klebsiella spp. 
Tabela 2

Perfil de sensibilidade dos microrganismos identificados nas uroculturas até 24 horas da retirada da sonda vesical em mulheres submetidas a cirurgias ginecológicas em hospital escola do nordeste do Brasil em 2007.

\begin{tabular}{lcccccc}
\hline Antimicrobianos & \multicolumn{2}{c}{ Klebsiella spp. } & \multicolumn{2}{c}{ Escherichia coli } & Outros Gram-negativos \\
\cline { 2 - 7 } & $\%{ }^{*}$ & Total** & $\% *$ & Total** & $\%$ Total** \\
\hline Ácido nalidíxico & 81,8 & 22 & 72,7 & 11 & 83,3 & 5 \\
Amicacina & 95,5 & 22 & 100,0 & 12 & 100,0 & 8 \\
Ampicilina & 31,8 & 22 & 8,3 & 12 & 50,0 & 8 \\
Cefalotina & 33,3 & 22 & 25,0 & 12 & 30,8 & 8 \\
Cefepima & 86,4 & 22 & 100,0 & 12 & 100,0 & 8 \\
Cefotaxima & 86,4 & 22 & 100,0 & 11 & 100,0 & 4 \\
Cefoxitina & 77,3 & 22 & 100,0 & 11 & 60,0 & 5 \\
Ceftazidima & 86,4 & 22 & 100,0 & 11 & 100,0 & 5 \\
Ceftriaxona & 86,4 & 22 & 100,0 & 11 & 100,0 & 5 \\
Ciprofloxacina & 95,5 & 22 & 75,0 & 12 & 87,5 & 8 \\
Gentamicina & 81,8 & 22 & 66,7 & 12 & 75,0 & 8 \\
Meropenem & 100,0 & 22 & 100,0 & 11 & 100,0 & 6 \\
Nitrofurantoína & 22,7 & 22 & 100,0 & 11 & 37,5 & 8 \\
Piperacilina-tazobactam & 100,0 & 19 & 100,0 & 11 & 100,0 & 5 \\
Sulfa-Trimetoprim & 77,3 & 22 & 25,0 & 12 & 75,0 & 8 \\
Ticarcilina-Clavulanato & 90,9 & 22 & 81,8 & 9 & 100,0 & 5 \\
\hline
\end{tabular}

* Percentual de sensibilidade; ** Número total de amostras de urina testadas quanto à sensibilidade para cada antimicrobiano.

rapia. O tempo médio de permanência da sonda vesical foi de 21 horas, variando de uma a 72 horas.

Quanto às características das mulheres submetidas a cirurgia ginecológica que apresentaram bacteriúria na coleta até 24 horas da retirada da sonda vesical, $47,8 \%(\mathrm{n}=22)$ tinham idade maior que 45 anos, 39,1\% $(\mathrm{n}=18)$ até cinco anos de estudo, $45,7 \%(\mathrm{n}=21)$ apresentavam número maior que três gestações e $37,0 \%(n=17)$ tinham paridade maior que três.

Entre as mulheres que apresentaram bacteriúria com sete/10 dias após sondagem vesical, 52,0\% $(\mathrm{n}=13)$ apresentaram idade maior que 45 anos, $60,0 \%(n=15)$ até cinco anos de estudo, 40,0\% $(\mathrm{n}=10)$ tinham número maior que três gestações e $28,0 \%(n=7)$ paridade maior que três.

Entre as 46 mulheres com urocultura positiva com até 24 horas da retirada da sonda vesical, os microrganismos mais encontrados foram Klebsiella spp. $(47,8 \% ; \mathrm{n}=22)$, seguido de E. coli $(26,1 \%$; $\mathrm{n}=12)$ e outros Gram-negativos $(17,4 \% ; \mathrm{n}=8)$ (Tabela 1). Nas uroculturas realizadas com sete/10 dias da sondagem vesical, as espécies bacterianas mais encontradas foram a Klebsiella spp. $(44,0 \%$; $\mathrm{n}=11)$, E. coli $(24,0 \% ; \mathrm{n}=6)$ e outros Gram-negativos $(20,0 \% ; n=5)$ (Tabela 1).

A sensibilidade antimicrobiana na urina coletada até 24 horas da retirada da sonda vesical para a Klebsiella spp. foi de $100 \%$ para o meropenem, $95,5 \%$ para a amicacina e ciprofloxacina, 90,9\% para a ticarcilina-clavulanato, $86,4 \%$ para a cefepima, cefotaxima, ceftazidima, ceftriaxona e piperacilina-tazobactam, $81,8 \%$ de sensibilidade para o ácido nalidíxico e gentamicina e 77,3\% para a cefoxitina e sulfametoxazol-trimetoprim (Tabela 2).

A E . coli apresentou uma sensibilidade na urina coletada com até 24 horas da retirada da sonda vesical de $100 \%$ para amicacina e cefepima, $91,7 \%$ para a cefotaxima, cefoxitina, cefotaxidina, ceftriaxona, meropenem, nitrofurantoína e piperacilinatazobactam, 83,3\% para a ticarcilina-clavulanato e 75,0\% para a ciprofloxacina (Tabela 2).

A sensibilidade antimicrobiana para urocultura com sete/10 dias após a sondagem vesical para a Klebsiella spp. foi de $100 \%$ para o ácido nalidixico, amicacina, cefotaxima, cefoxitina, ceftazidima, ceftriaxona, ciprofloxacina, gentamicina, meropenem e piperacilina-tazobactam e $90,9 \%$ para o cefepima, sulfametoxazol-trimetoprim e ticarcilinaclavulanato (Tabela 3 ).

A sensibilidade da $E$. coli aos antibióticos na urocultura com sete/10 dias foi de $100 \%$ para a amicacina, cefoxitina, ciprofloxacina, gentamicina e meropenem e $83,3 \%$ para a cefepima, cefotaxima, 
Perfil de sensibilidade dos microrganismos identificados nas uroculturas sete/10 dias da sondagem vesical em mulheres submetidas a cirurgias ginecológicas em um hospital escola do nordeste do Brasil em 2007.

\begin{tabular}{|c|c|c|c|c|c|c|}
\hline \multirow[t]{2}{*}{ Antimicrobianos } & \multicolumn{2}{|c|}{ Klebsiella spp } & \multicolumn{2}{|c|}{ Escherichia coli } & \multicolumn{2}{|c|}{ Outros Gram-negativos } \\
\hline & $\%$ * & Total** & $\%$ * & Total** & $\%$ * & Total** \\
\hline Ácido nalidíxico & 100,0 & 11 & 66,7 & 6 & 75,0 & 4 \\
\hline Amicacina & 100,0 & 11 & 100,0 & 6 & 100,0 & 4 \\
\hline Ampicilina & 18,2 & 11 & 33,3 & 6 & 25,0 & 4 \\
\hline Cefalotina & 72,7 & 11 & 50,0 & 6 & 50,0 & 4 \\
\hline Cefepima & 90,9 & 11 & 83,3 & 6 & 100,0 & 4 \\
\hline Cefotaxima & 100,0 & 11 & 83,3 & 6 & 100,0 & 4 \\
\hline Cefoxitina & 100,0 & 11 & 100,0 & 6 & 75,0 & 4 \\
\hline Ceftazidima & 100,0 & 11 & 83,3 & 6 & 100,0 & 4 \\
\hline Ceftriaxona & 100,0 & 11 & 83,3 & 6 & 100,0 & 4 \\
\hline Ciprofloxacina & 100,0 & 11 & 100,0 & 6 & 100,0 & 4 \\
\hline Gentamicina & 100,0 & 11 & 100,0 & 6 & 100,0 & 4 \\
\hline Meropenem & 100,0 & 11 & 100,0 & 6 & 100,0 & 4 \\
\hline Nitrofurantoína & 45,5 & 11 & 83,3 & 6 & 75,0 & 4 \\
\hline Piperacilina-tazobactam & 100,0 & 11 & 83,3 & 6 & 100,0 & 4 \\
\hline Sulfa-Trimetoprim & 90,9 & 11 & 83,3 & 6 & 50,0 & 4 \\
\hline Ticarcilina-Clavulanato & 90,9 & 11 & 83,3 & 6 & 75,0 & 4 \\
\hline
\end{tabular}

* Percentual de sensibilidade; ** Número total de amostras de urina testadas quanto à sensibilidade para cada antimicrobiano.

Tabela 4

Via de administração e sensibilidade antimicrobiana de 75,0\% ou mais nas uroculturas até 24 horas depois da retirada da sonda e sete/10 dias após sondagem vesical em mulheres submetidas a cirurgias ginecológicas em um hospital escola do nordeste do Brasil em 2007.

\begin{tabular}{|c|c|c|c|c|c|c|c|}
\hline \multirow[t]{3}{*}{ Antimicrobianos } & \multicolumn{6}{|c|}{ Sensibilidade antimicrobiana $\geq 75 \%$} & \multirow[t]{3}{*}{ Via de administração* } \\
\hline & \multicolumn{2}{|c|}{ Klebsiella spp } & \multicolumn{2}{|c|}{ E. coli } & \multicolumn{2}{|c|}{$\begin{array}{l}\text { Outros Gram- } \\
\text { negativos }\end{array}$} & \\
\hline & $24 \mathrm{~h}$ & $7 / 10$ dias & $24 \mathrm{~h}$ & $7 / 10$ dias & $24 \mathrm{~h}$ & $7 / 10$ dias & \\
\hline Ácido nalidíxico & $x$ & $x$ & & & & & Oral \\
\hline Amicacina & $x$ & $x$ & $x$ & $x$ & $x$ & $x$ & Parenteral \\
\hline Cefepima & $\mathrm{x}$ & $\mathrm{x}$ & $\mathrm{x}$ & $\mathrm{x}$ & $\mathrm{x}$ & $\mathrm{x}$ & Parenteral \\
\hline Cefotaxima & $x$ & $x$ & $x$ & $x$ & & $x$ & Parenteral \\
\hline Cefoxitina & $\mathrm{x}$ & $\mathrm{x}$ & $\mathrm{x}$ & $\mathrm{x}$ & & $\mathrm{x}$ & Parenteral \\
\hline Ceftazidima & $x$ & $x$ & $x$ & $x$ & & $x$ & Parenteral \\
\hline Ceftriaxona & $x$ & $x$ & $\mathrm{x}$ & $\mathrm{x}$ & & $\mathrm{x}$ & Parenteral \\
\hline Ciprofloxacina & $x$ & $x$ & $x$ & $x$ & $x$ & $x$ & Oral e Parenteral \\
\hline Gentamicina & $x$ & $\mathrm{x}$ & & $x$ & $x$ & $x$ & Parenteral \\
\hline Meropenem & $x$ & $x$ & $\mathrm{x}$ & $x$ & $x$ & $x$ & Parenteral \\
\hline Nitrofurantoína & & & $x$ & $x$ & & $x$ & Oral \\
\hline Piperacilina-tazobactam & $x$ & $x$ & $x$ & $x$ & & $x$ & Parenteral \\
\hline Sulfa-Trimetoprim & $x$ & $x$ & & $x$ & $x$ & & Oral \\
\hline Ticarcilina-Clavulanato & $x$ & $x$ & $x$ & $x$ & $x$ & $x$ & Parenteral \\
\hline
\end{tabular}

* Fonte: Dicionário de especialidades farmacêuticas. Rio de Janeiro; 2008.32 
ceftazidima, ceftriaxona, nitrofurantoína, piperacilina-tazobactam, sulfametoxazol-trimetoprim e ticarcilina-clavulanato (Tabela 3).

Os antimicrobianos que apresentaram boa sensibilidade, maior ou igual a $75 \%$, nas duas uroculturas para as bactérias mais freqüentes (Klebsiella spp., E. coli e outros Gram-negativos) foram amicacina, cefepima, ceftazidima, ciprofloxacina, meropenem e ticarcilina-clavulonato, dos quais apenas a ciprofloxacina pode ser administrada por via oral (Tabela 4).

\section{Discussão}

A incidência de infecção urinária encontrada na literatura após cateterismo vesical varia de 10 a 20\%.8,15,23 Entretanto, neste estudo, a freqüência de bacteriúria após cateterização vesical nas cirurgias ginecológicas foi de $23,6 \%$ com a coleta de urina realizada com até 24 horas da retirada da sonda vesical e de $11,1 \%$ com sete/10 dias da sondagem vesical, sendo que $2,4 \%$ das mulheres apresentaram sintomatologia urinária. Essas taxas estão acima das descritas na literatura sobre ITU relacionada à cateterização. 14,24 Ressalta-se, todavia, que esses estudos não incluíram apenas cirurgias ginecológicas e que essas variações na freqüência de bacteriúria podem estar relacionadas aos fatores de risco, ao setor em que foi realizado o estudo e ao tempo que foi coletado a urina. Enquanto, no presente estudo, a urina foi coletada com até 24 horas depois de retirada a sonda e com sete/10 dias após a sondagem vesical, em outros trabalhos coletou-se a urina apenas após a retirada imediata da sonda vesical.11,16,23

Sabe-se que a incidência de ITU aumenta com o tempo de permanência da sonda vesical no trato urinário. ${ }^{14,17}$ Entretanto, nessa pesquisa, o tempo médio de permanência com a sonda vesical foi de 21 horas, tempo considerado baixo para elevar as taxas de bacteriúria. 11,16,23

Nos últimos anos, tem-se demonstrado uma tendência a mudanças na etiologia das infecções do trato urinário. Os microrganismos Gram-positivos estão sendo paulatinamente mais isolados nas uroculturas do que a $E$. coli, o que pode ser atribuído ao uso indiscriminado de antibióticos com eficácia limitada contra os patógenos Gram-positivos, permitindo assim sua proliferação. 25 Por esse motivo, é importante que cada clínica possa avaliar quais os microrganismos mais encontrados nas bacteriúrias, possibilitando uma terapêutica mais adequada, quando necessária. Entretanto, a E. coli ainda é o agente etiológico mais freqüentemente isolado nas uroculturas. 7,9,26 Ressalta-se que tais estudos não foram realizados em pacientes submetidas a cirurgia ginecológica com sondagem vesical.

Em relação aos principais microrganismos isolados nas uroculturas, comparou-se a freqüência desses na coleta até 24 horas da retirada da sonda com a de sete/10 dias da sondagem vesical, não sendo encontrada nenhuma diferença significativa entre os microrganismos. Foi observado que os Gram-negativos foram os mais freqüentes, como a Klebsiella spp. $(47,8 \% \times 44,0 ; p=0,76)$, a E. coli $(26,1 \% \times 24,0 ; p=0,85)$ e outros Gram-negativos $(17,4 \% \times 20,0 ; p=0,51)$. Provavelmente esse achado se deve ao controle e às normas da CCIH para um uso mais racional dos antibióticos, 27 o que dificulta, assim, o aumento da resistência bacteriana. Porém, é importante ressaltar que as uroculturas positivas das mulheres estudadas tiveram, em sua maior parte, resoluções espontâneas, sem necessidade de tratamento específico, e apenas seis pacientes tiveram bacteriúria sintomática necessitando de terapêutica.

Semelhante a este estudo, descreve-se na literatura uma elevada freqüência de resistência microbiana aos antibióticos ampicilina e sulfametoxazoltrimetoprim.28,29 Entretanto, em alguns países existem relatos do surgimento de resistência às fluoroquinolonas, 30,31 o que não foi observado nos resultados do presente estudo. A distribuição dos patógenos causadores das infecções, especialmente os com resistência antimicrobiana, varia com o tempo e os hospitais em diferentes regiões do mundo. 25 Daí a necessidade de cada serviço avaliar o perfil microbiano e a sensibilidade antimicrobiana para as diversas infecções hospitalares.

Nas uroculturas realizadas, a Klebsiella spp. foi o agente mais freqüente, com maior sensibilidade antimicrobiana (acima de 75\%) para o ácido nalidixico, amicacina, cefepima, cefotaxima, ceftazidima, ceftriaxona, ciprofloxacina, gentamicina, piperacilina-tazobactam e ticarcilina-clavulanato. Foi observada resistência acima de 50\% para ampicilina, cefalotina, cloranfenicol e nitrofurantoína. Esses achados são importantes para que os órgãos controladores de infecção da instituição monitorizem o uso racional dos antimicrobianos e a microbiota existente. A resistência apresentada pela Klebsiella spp. para ampicilina (77,7\%), sulfametoxazol/trimetoprim $(46,6 \%)$, nitrofurantoína $(24 \%)$ e ciprofloxacino (24\%) já foi relatada. 26

Os Gram-positivos tiveram freqüências bem inferiores ao esperado, não atingindo nem $20 \%$ dos casos. Esses dados reiteram a importância de se 
determinar a microbiota e o seu perfil de sensibilidade em cada serviço. 25

O Council for Appropriate and Racional Antibiotic Therapy (CARAT) chama atenção para a utilização indiscriminada de antibióticos, sugerindo estabelecer normas para avaliação criteriosa na escolha do tratamento, devendo ser baseado em evidências clínicas para uma terapêutica segura, eficaz e uma adequada relação custo-benefício. 25 Analisando os resultados aqui apresentados à luz dessas recomendações, os antimicrobianos que apresentaram sensibilidade maior ou igual a $75 \%$ nas duas uroculturas, para as bactérias mais freqüentes

\section{Referências}

1. Stamm WE. Scientific and clinical challenges in the management of urinary tract infections. Am J Med. 2002; 113: $15-45$.

2. McLaughlin SP, Carson CC. Urinary tract infection in women. Med Clin N Am. 2004; 88: 417-29.

3. Foxman B. Epidemiology of urinary tract infection: incidence, morbidity, and economic cost. Am J Med. 2002; 113 (Suppl 1A): 5S-13S.

4. Gonzalez CM, Schaeffer AJ. Treatment of urinary tract infection: what's old, what's new, and what works. World J Urol. 1999; 17: 372-82.

5. NCCLS (National Committee for Clinical Laboratory Standards). Performance standards for antimicrobial disk susceptibility testing. Wayne, PA; 2004. [Fourteenth informational supplement, M100-S14].

6. Mohanty S, Xess I, Hasan F, Kapil A, Mittal S, Tolosa JE. Prevalence \& susceptibility to fluconazole of Candida species causing vulvovaginitis. Indian J Med Res. 2007; 126: $216-9$.

7. Heilberg IP, Schor N. Abordagem diagnóstica e terapêutica na infecção do trato urinário-ITU. Rev Assoc Med Bras. 2003; 49:1 09-16.

8. Gokula RR, Hickner JA, Smith MA. Inappropriate use of urinary catheters in elderly patients at a midwestern community teaching hospital. Am J Infect Control. 2004; 32: $196-9$.

9. Savas L, Guvel S, Onlen Y, Savas N, Duran N. Nosocomial urinary tract infections: micro-organisms, antibiotic sensitivities and risk factors. West Indian Med J. 2006; 55: 18893.

10. Hart S. Aseptic technique. In: Dougherty L, Lister S, editors. The Royal Marsden Hospital: manual of clinical nursing procedures. 6. ed. Oxford: Blackwell; 2004. p. 5063.
(Klebsiella spp., E. coli e outros Gram-negativos) foram amicacina, cefepima, ceftazidima, ciprofloxacina, meropenem e ticarcilina-clavulonato. $\mathrm{O}$ antibiótico com sensibilidade maior ou igual a $95 \%$ para Klebsliella spp. e $75 \%$ para E. coli foi a ciprofloxacina, apresentando a vantagem da via de administração oral. 32

Diante da maior sensibilidade apresentada pelos microrganismos isolados na segunda amostra (sete/10 dias), fica caracterizado que a bacteriúria nesses casos não se trata de infecção hospitalar, mas evento inerente ao procedimento, sugerido pela resolução espontânea da maioria das infecções.
11. Niël-Weise BS, Broek PJ. Urinary catheter policies for short-term bladder drainage in adults [Cochrane review]. Cochrane Library (4); 2007.

12. Griffiths R, Fernandez R. Strategies for the removal of short-term indwelling urethral catheters in adults [Cochrane review]. Cochrane Library (4); 2007.

13. Sedor J, Mulholland SG. Hospital-adquired urinary tract infections associated with the indewelling catheter. Urol Clin Morth Am. 1999; 26: 821-28.

14. Getliffe K. Care of urinary catheters. Nur Stand. 1996; 11: 47-50.

15. Tambyah PA, Maki DG. Catheter-associated urinary tract infection is rarely symptomatic: a prospective study of 1,497 catheterized patients. Arch Intern Med. 2000; 160: 678-82.

16. Schiotz HA, Tanbo TG. Postoperative voiding, bacteriuria and urinary tract infection with Foley catheterization after gynecological surgery. Acta Obstet Gynecol Scand. 2006; 85: 476-81.

17. Graves N, Tong E, Morton AP, Halton K, Curtis M, Lairson D, Whitby M. Factors associated with health care-acquired urinary tract infection. Am J Infect Control. 2007; 35: 38792.

18. Ronald A. The etiology of urinary tract infection: traditional and emerging pathogens. Am J Med. 2002; 113 (Suppl 1A): 14S-19S.

19. Niclle LE. Epidemiology of urinary tract infection. Infect Med. 2001; 18: 153-62.

20. Verhaz A, Skrbic R, Rakita-Music M. Resistance of catheter-associated urinary tract infections to antibacterials. Vojnosanit Pregl. 2005; 62: 181-7.

21. O’Donnell JA, Hofmann MT. Urinary tract infection. How to manage nursing home pacients with or without chronic cathterization. Geriatrics. 2002; 57: 45-58. 
22. Bauer AW, Kirby EM. Antibiotic susceptibility testing by standardized single disk method. Am J Clin Pathol. 1996; 45:493-6.

23. Stamm AMNF, Coutinho MSSA. Infecção do trato urinário relacionada ao cateter vesical de demora: incidência e fatores de risco. Rev Assoc Med Bras. 1999; 45: 27-33.

24. Maki DG, Tambyah PA. Engineering out the risk of infection with urinary catheters. Emerg Infect Dis. 2004; 7: 2-5.

25. Liu H, Mulholland SG. Appropriate antibiotic treatment of genitourinary infections in hospitalized patients. Am J Med. 2005; 118 (Suppl 7A): 14S-20S.

26. Blatt JM, Miranda MC. Perfil dos microorganismos causadores de infecções do trato urinário em pacientes internados. Rev Panam Infectol. 2005; 7: 10-14.

27. Brasil. Ministério da Saúde. Portaria n. 2.616, de 12 de maio de 1998. Estabelece diretrizes e normas para a prevenção e controle das infecções hospitalares. Diário Oficial da União [DOU]. Brasília, DF; 13 maio 1998; Seção 1, p. 133.

28. Winstanley TG, Limb DI, Eggington R, Hancock F. A 10 year survey of the antimicrobial susceptibility of urinary tract isolates in the UK: the microbe base project. J Antimicrob Chemother. 1997; 40: 591-4.

29. Trienekens T, Stobberingh E, Beckers F, Knottnerus A. The antibiotic susceptibility patterns do uropathogens isolated from general pratice patients in southern Netherlands. J Antimicrob Chemother. 1994; 33: 1064-6.

30. Garau J, Xercavins M, Rodriguez-Carballeria M, GomesVera JR, Coll I, Vidal D, Llovet T, Ruíz-Bremón A. Emergence and dissemination of quinolone-resistant Escherichia coli in the community. Antimicrob Agents Chemother. 1999; 43: 2736-41.

31. Aguiar JM, Chacon J, Canton R, Baquero F. The emergence of highly fluoroquinolone-resistant Eschenchia coli in community-acquired urinary tract infections. J Antimicrob Chemother. 1992; 29: 349-50.

32. Dicionário de Especialidades Farmacêuticas: DEF 2007/08. 36. ed. Rio de Janeiro: Publicações Científicas; 2008. p. 930 .

Recebido em 22 de fevereiro de 2008

Versão final apresentado em 25 de setembro de 2008

Aprovado em 5 de outubro de 2008 